\title{
Água levemente salinizada aumenta a eficiência da larvicultura de peixes neotropicais
}

\author{
Rosângela Kiyoko Jomori(1), Ronald Kennedy Luz ${ }^{(2)}$, Rodrigo Takata(2), Thiago El Hadi Perez Fabregat( ${ }^{(3)}$ \\ e Maria Célia Portella(4)
}

\begin{abstract}
(1)Fundação Educacional de Ituverava, Faculdade Dr. Francisco Maeda, Laboratório de Aquicultura, Rodovia Jerônimo Nunes Macedo Km 01, CEP 14500-000, Ituverava, SP. E-mail: jomori@netsite.com.br (2)Universidade Federal de Minas Gerais, Escola de Veterinária, Laboratório de Aquacultura, Avenida Antônio Carlos, no 6.627, CEP 31270-901, Belo Horizonte, MG. E-mail: luzrk@yahoo.com, rodrigo.takata@bol.com.br (3)Universidade do Estado de Santa Catarina, Centro de Ciências Agroveterinárias, Avenida Luiz de Camões, no 2.090, Bairro Conta Dinheiro, CEP 88520-000 Lages, SC. E-mail: thiagofabregat@hotmail.com ${ }^{(4)}$ Universidade Estadual Paulista, Centro de Aquicultura, Via de Acesso Prof. Paulo Donato Castellane, s/nº, CEP 14884-900, Jaboticabal, SP. E-mail: portella@caunesp.unesp.br
\end{abstract}

Resumo - O objetivo deste trabalho foi avaliar o efeito de água levemente salinizada sobre a larvicultura intensiva do tambaqui, matrinxã, apaiari e piau, durante os dias iniciais de alimentação. As larvas foram mantidas em água artificialmente salinizada, em concentrações de 0 (água doce) a $14 \mathrm{~g} \mathrm{~L}^{-1}$ de $\mathrm{NaCl}$ (intervalo de 2,0 $\mathrm{g} \mathrm{L}^{-1}$ ), e foram alimentadas com duas porções diárias de náuplios de Artemia, de acordo com protocolo para cada espécie, em delineamento inteiramente casualizado, com três repetições. Larvas de tambaqui, matrinxã e apaiari podem ser cultivadas em até $2 \mathrm{~g} \mathrm{~L}^{-1}$ de concentração salina, sem prejuízos ao crescimento e à sobrevivência. Larvas de piau foram mais tolerantes e suportaram até $4 \mathrm{~g} \mathrm{~L}^{-1}$ de concentração salina. Acima disto, a mortalidade dos peixes aumentou e chegou a $100 \%$ à concentração de $6 \mathrm{~g} \mathrm{~L}^{-1}$ (matrinxã e apaiari) e de $10 \mathrm{~g} \mathrm{~L}^{-1}$ (tambaqui). A salinização da água a $2 \mathrm{~g} \mathrm{~L}^{-1}$ proporcionou maior taxa de sobrevivência a larvas de matrinxã e maior crescimento a larvas de tambaqui, apaiari e piau. Estas duas últimas espécies apresentaram melhor crescimento com a maior quantidade de náuplios. Água salinizada a $2 \mathrm{~g} \mathrm{~L}^{-1}$ é benéfica para as espécies estudadas, pois otimiza o potencial de crescimento das larvas e o uso de náuplios de Artemia como alimento vivo.

Termos para indexação: Astronotus ocellatus, Brycon amazonicus, Colossoma macropomum, Leporinus macrocephalus, alimento vivo, náuplios de Artemia.

\section{Slightly saline water increases the larviculture efficiency of freshwater Neotropical fish}

\begin{abstract}
The objective of this work was to evaluate the effect of slightly saline water in an intensive larviculture of tambaqui, matrinxã, apaiari, and piau, during the initial days of active feeding. Larvae were reared in artificial saline water, at concentrations from 0 (freshwater) to $14 \mathrm{~g} \mathrm{~L}^{-1} \mathrm{NaCl}$ (at a $2.0 \mathrm{~g} \mathrm{~L}^{-1}$ ), and fed with two portions of Artemia nauplii, following a specific protocol for each species, in a completely randomized design, with 3 replicates. Tambaqui, matrinxã and apaiari larvae can be reared at saline concentrations till $2 \mathrm{~g} \mathrm{~L}^{-1}$ without impairing growth and survival. Piau larvae were more tolerant and endured saline water until $4 \mathrm{~g} \mathrm{~L}^{-1}$. At saline concentrations above that, fish mortality increased and reached $100 \%$ at $6 \mathrm{~g} \mathrm{~L}^{-1}$, for matrinxã and apaiari, and at $10 \mathrm{~g} \mathrm{~L}^{-1}$, for tambaqui. Saline water at $2 \mathrm{~g} \mathrm{~L}^{-1}$ provided a higher survival for matrinxã, and higher growth for tambaqui, apaiari and piau. These last two species showed a better growth performance with the highest quantity of nauplii. Slightly saline water at $2 \mathrm{~g} \mathrm{~L}^{-1}$ is beneficial to these studied species, as it optimizes larval growth potential and the use of Artemia nauplii as live food.

Index terms: Astronotus ocellatus, Brycon amazonicus, Colossoma macropomum, Leporinus macrocephalus, live food, Artemia nauplii.
\end{abstract}

\section{Introdução}

A larvicultura de peixes neotropicais tem-se aprimorado com a adoção do sistema intensivo, como técnica de criação de larvas e juvenis (Portella \& Dabrowski, 2008), seguindo a tendência dos sistemas adotados em outros países para produção de peixes marinhos (Conceição et al., 2010). A alimentação é um dos fatores mais relevantes durante a larvicultura (Feiden et al., 2006), e é definitiva para garantir o desenvolvimento e a sobrevivência dos animais nesta fase. Assim como as larvas de peixes marinhos, as das 
espécies neotropicais mais importantes na aquicultura sul-americana apresentam características altriciais e dependem de suprimento de alimento vivo durante a fase inicial de alimentação exógena (Portella \& Dabrowski, 2008). Entre os diferentes organismos-alimento mais utilizados, os náuplios de Artemia têm mostrado que são eficientes em promover o desenvolvimento e o crescimento das larvas de peixes de água doce criadas intensivamente (Kaiser et al., 2003; Luz \& Santos, 2010; Jomori et al., 2012).

Os Anostraca do gênero Artemia são microcrustáceos de ambientes salinos que, em determinadas condições, produzem ovos de resistência denominados cistos. Os cistos nacionais ou importados são facilmente adquiridos no mercado, pois os náuplios constituem um importante insumo para várias atividades de aquicultura, sobretudo para a criação de larvas de peixes e de camarões marinhos e de água doce. No entanto, na larvicultura de água doce, um dos inconvenientes é o fato de os náuplios de Artemia apresentarem tempo de vida limitado nesse ambiente. Consequentemente, a morte dos náuplios em poucas horas restringe o tempo em que ficam disponíveis às larvas, além de favorecer a deterioração da água (Beux \& Zaniboni-Filho, 2006; Jomori et al., 2012). Essa limitação tem levado ao desenvolvimento da larvicultura de peixes de água doce em ambientes ligeiramente salinizados (Luz \& Portella, 2002; Weingartner \& Zaniboni Filho, 2004; Beux \& Zaniboni Filho, 2007; Jomori et al., 2012), que possibilitam maior longevidade aos náuplios (Jomori et al., 2012). Além disso, o sal também é benéfico para prevenir doenças (Altinok \& Grizzle, 2001; Garcia et al., 2007; Souza-Bastos \& Freire, 2009), reduzir a diferença osmótica entre o animal e o meio externo e reduzir o estresse (Wurts, 1995).

Outro fator importante que afeta o crescimento das larvas de peixes é a quantidade de alimento ofertada diariamente (Rabe \& Brown, 2000). O estabelecimento de protocolos de alimentação adequados contribui para diminuir a mortalidade (Conceição et al., 2010) e permite melhor programação dos ciclos de produção. Hipoteticamente, para as larvas de peixes de água doce, a disponibilidade de náuplios de Artemia pode ser influenciada pelo procedimento da salinização da água, estendendo o tempo de vida dos náuplios e favorecendo, assim, maiores taxas de encontro e de ingestão dos mesmos pelas larvas.

O objetivo deste trabalho foi avaliar o efeito de água levemente salinizada sobre a larvicultura intensiva do tambaqui, matrinxã, apaiari e piau, durante os dias inicias de alimentação.

\section{Material e Métodos}

O estudo consistiu de quatro experimentos, realizados no Centro de Aquicultura da Universidade Estadual Paulista (Caunesp), Jaboticabal, SP, no período reprodutivo de novembro de 2002 a fevereiro de 2003.

Foram estudadas as seguintes espécies neotropicais: tambaqui (Colossoma macropomum), matrinxã (Brycon amazonicus), apaiari (Astronotus ocellatus) e piau (Leporinus macrocephalus). A incubação dos ovos e a manutenção das larvas destas espécies, durante o período endotrófico, foram realizadas em água doce. Logo após o início da alimentação exógena, as larvas foram transferidas diretamente para os ambientes salinizados.

As unidades experimentais foram mantidas em sistema de banho termostatizado, para estabilização da temperatura $\left(29,0 \pm 1,0^{\circ} \mathrm{C}\right)$, e com aeração artificial constante para o suprimento de oxigênio dissolvido (> 4,5 $\left.\mathrm{mg} \mathrm{L}^{-1}\right)$. A água utilizada provinha de poço

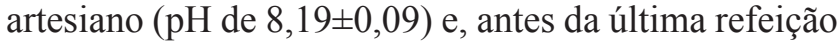
diária, os detritos acumulados no fundo das unidades experimentais eram sifonados. Nesse momento, cerca de $90 \%$ do volume da água era renovado com água à temperatura semelhante e com as respectivas salinidades. O preparo da água salinizada foi feito diariamente e por meio de adição de cloreto de sódio comercial, de origem marinha. As salinidades foram aferidas com o auxílio de sonda multiparamétrica YSI modelo 550A (YSI Inc. Yellow Springs, OH, USA). O fotoperíodo foi de 10 horas de luz.

As larvas das quatro espécies foram alimentadas com náuplios de Artemia, obtidos diariamente pela incubação dos cistos em água salinizada a $10 \mathrm{~g} \mathrm{~L}^{-1}$. $\mathrm{O}$ alimento foi oferecido em três refeições diárias (às 8:00, 13:00 e 17:00 horas), em quantidades específicas para cada espécie, baseadas em estudos preliminares realizados no mesmo laboratório.

Após a introdução das larvas nos meios salinizados, foram realizadas observações a cada duas horas, aproximadamente, nas primeiras 96 horas do experimento, para acompanhar a mortalidade dos animais resultante da transferência e do choque salino. 
O crescimento das larvas foi avaliado pelas medidas de comprimento total $(\mathrm{mm})$ e massa úmida individual (mg), aferidos no início e no final de cada experimento, quando também foram determinadas as taxas de sobrevivência.

\section{Larvicultura do tambaqui (Colossoma macropomum)}

Larvas de tambaqui, com quatro dias de vida (comprimento total de $6,00 \pm 0,16 \mathrm{~mm}$ e massa úmida de $0,42 \pm 0,16 \mathrm{mg}$ ), foram transferidas diretamente da água doce para as concentrações salinizadas a (S): 0 , 2, 4, 6, 8, 10, 12 e $14 \mathrm{~g}$ de $\mathrm{NaCl} \mathrm{L}^{-1}$. As quantidades diárias de alimento foram de 500 náuplios por larva, durante os primeiros cinco dias, e de 750 náuplios por larva do sexto ao décimo dia.

As larvas foram estocadas à densidade de $40 \mathrm{~L}^{-1}$, em 24 unidades experimentais com $1 \mathrm{~L}$ de água cada uma. $\mathrm{O}$ experimento foi realizado em delineamento inteiramente casualizado, com oito tratamentos e três repetições, por 10 dias.

$\mathrm{O}$ experimento com as larvas de tambaqui foi o primeiro dos quatro do presente trabalho. Com base nos resultados deste experimento, verificou-se a importância de investigar conjuntamente a quantidade de náuplios de Artemia disponível às larvas na coluna d'água. Assim, além da salinidade, duas quantidades diárias de alimento foram associadas aos protocolos experimentais dos três ensaios seguintes.

\section{Larvicultura do matrinxã (Brycon amazonicus)}

Larvas de matrinxã, com 28 horas de vida, foram estocadas à densidade de $15 \mathrm{~L}^{-1}$, em 24 unidades experimentais com $5 \mathrm{~L}$ de água cada uma. Às 32 horas de vida $(5,54 \pm 0,29 \mathrm{~mm}$ de comprimento total e $0,85 \pm 0,16 \mathrm{mg}$ de massa úmida), quando já apresentavam a boca aberta, as larvas começaram a receber o alimento. As salinidades $(\mathrm{S})$ testadas foram de: $0,2,4 \mathrm{e} 6 \mathrm{~g}$ de $\mathrm{NaCl} \mathrm{L}^{-1}$. Neste experimento, testou-se até a salinidade de $6 \mathrm{~g} \mathrm{~L}^{-1}$, em razão da disponibilidade de animais. Para cada salinidade, foram testadas as quantidades diárias $(\mathrm{Q})$ de alimento com náuplios de Artemia: $\mathrm{Q}_{1}=1.250$ náuplios por larva e $\mathrm{Q}_{2}=2.500$ náuplios por larva. $\mathrm{O}$ experimento teve duração de cinco dias, período considerado crítico para a espécie, pela alta incidência de canibalismo.

$\mathrm{O}$ experimento foi realizado em delineamento inteiramente casualizado, em arranjo fatorial $4 \times 2$ (quatro concentrações salinas e duas quantidades diárias de presas), com três repetições.

\section{Larvicultura do apaiari (Astronotus ocellatus)}

Larvas de apaiari, com três dias de vida (comprimento total de $6,92 \pm 0,36 \mathrm{~mm}$ e massa de $3,75 \pm 0,58 \mathrm{mg}$ ), foram estocadas à densidade de $4 \mathrm{~L}^{-1}$, em 48 unidades experimentais com $5 \mathrm{~L}$ de água cada uma. As larvas foram transferidas da água doce para as salinidades de $0,2,4,6,8,10,12$ e $14 \mathrm{~g}_{\text {de }} \mathrm{NaCl} \mathrm{L}^{-1}$. Duas quantidades diárias de alimento foram testadas $\left(\mathrm{Q}_{1}\right.$ e $\left.\mathrm{Q}_{2}\right)$, com acréscimos do número de náuplios durante o experimento, em razão do crescimento do animal, com: $\mathrm{Q}_{1}=500$ e $\mathrm{Q}_{2}=1.000$ náuplios por larva, durante os primeiros cinco dias; $\mathrm{e}_{1}=2.000 \mathrm{e}$ $\mathrm{Q}_{2}=4.000$ náuplios por larva, do sexto ao décimo dia, em adaptação do protocolo estabelecido para pacu por Jomori et al. (2005).

$\mathrm{O}$ experimento foi realizado em delineamento inteiramente casualizado, em arranjo fatorial $8 \times 2$ (oito concentrações salinas e duas quantidades diárias de alimento), com três repetições, durante dez dias.

\section{Larvicultura do piau (Leporinus macrocephalus)}

Larvas de piau, com três dias de vida $(4,50 \pm 0,40 \mathrm{~mm}$ de comprimento total e $0,32 \pm 0,10 \mathrm{mg}$ de massa), foram estocadas à densidade de $40 \mathrm{~L}^{-1}$, em 24 unidades experimentais com $1 \mathrm{~L}$ de água cada uma, às concentrações salinas (S) de 0,2 e $4 \mathrm{~g}$ de $\mathrm{NaCl} \mathrm{L}^{-1}$. As quantidades diárias $\left(\mathrm{Q}_{1}\right.$ e $\left.\mathrm{Q}_{2}\right)$ de náuplios de Artemia foram de $\mathrm{Q}_{1}=100$ e $\mathrm{Q}_{2}=200$ náuplios por larva, para os três primeiros dias, com aumento em dobro a cada três dias em: 200, 400 e 800 náuplios por larva, para $\mathrm{Q}_{1}$; e 400, 800 e 1600 náuplios por larva para $\mathrm{Q}_{2}$, no período de doze dias de larvicultura. $\mathrm{O}$ experimento foi realizado em arranjo fatorial $3 \times 2$ (três concentrações salinas e duas quantidades diárias de alimento), com três repetições.

As análises estatísticas foram realizadas pelo programa SAS versão 9.0. Os dados foram analisados quanto à normalidade e homogeneidade das variâncias, e os dados em percentuais foram transformados $\left[\mathrm{y}=\operatorname{arcsen}(\mathrm{x} / 100)^{0,5}\right]$ antes das análises, mas somente os dados percentuais são apresentados. Os resultados foram comparados estatisticamente pela análise de variância "one way" (experimento para larvicultura do tambaqui) e análise de variância fatorial (demais experimentos). Em caso de diferença significativa, as 
médias foram comparadas pelo teste de Tukey, a 5\% de probabilidade.

\section{Resultados e Discussão}

A salinização da água afetou o desempenho dos peixes, durante a larvicultura intensiva, das quatro espécies estudadas. Larvas de tambaqui e de apaiari apresentaram as maiores taxas de sobrevivência em água doce e na levemente salinizada a $2 \mathrm{~g} \mathrm{~L}^{-1}$ (Tabelas 1 e 2, respectivamente). As larvas de matrinxã apresentaram maior sobrevivência à concentração de $2 \mathrm{~g} \mathrm{~L}^{-1}$ (Tabela 2). As larvas de piau foram as mais tolerantes entre as espécies estudadas e não tiveram a sobrevivência prejudicada com o aumento da salinidade de 2 para $4 \mathrm{~g} \mathrm{~L}^{-1}$.

A mortalidade das larvas de matrinxã começou 24 horas após a transferência da água doce para a salinidade de $6 \mathrm{~g} \mathrm{~L}^{-1}$, e a mortalidade total (100\%) ocorreu após quatro dias de experimento. A mortalidade total de larvas de apaiari foi constatada à salinidade de $8 \mathrm{~g} \mathrm{~L}^{-1}$, após 48 horas de exposição. À concentração salina de $6 \mathrm{~g} \mathrm{~L}^{-1}$, larvas sobreviventes (cerca de 5\%) foram observadas em apenas uma das repetições de cada tratamento $\left(\mathrm{S}_{6} \mathrm{Q}_{1}\right.$ e $\left.\mathrm{S}_{6} \mathrm{Q}_{2}\right)$. Larvas de tambaqui suportaram salinidades de até $8 \mathrm{~g} \mathrm{~L}^{-1}$; entretanto, a taxa média de sobrevivência diminuiu com 0 aumento da salinidade de 4 para $8 \mathrm{~g} \mathrm{~L}^{-1}$ (Tabela 1). Recentemente, observou-se que larvas de pacu Piaractus mesopotamicus, espécie da mesma família que o tambaqui, suportam salinidades de até $8 \mathrm{~g} \mathrm{~L}^{-1} \mathrm{e}$, semelhantemente ao observado no tambaqui, as taxas de sobrevivência diminuíram de 73,0\% $\left(4 \mathrm{~g} \mathrm{~L}^{-1}\right)$ para $39,9 \%\left(8 \mathrm{~g} \mathrm{~L}^{-1}\right)$, em relação à $100 \%$ da sobrevivência constatada a $2 \mathrm{~g} \mathrm{~L}^{-1}$ (Jomori et al., 2012). A tolerância à

Tabela 1. Valores médios de comprimento total, massa e sobrevivência das larvas de tambaqui (Colossoma macropomum), após os primeiros 10 dias de alimentação ativa, com 500 náuplios de Artemia por larva durante os primeiros cinco dias, e 750 náuplios por larva do sexto ao décimo $\operatorname{dia}^{(1)}$.

\begin{tabular}{lrrrrr}
\hline Variável & \multicolumn{5}{c}{ Salinidade $\left(\mathrm{g} \mathrm{L}^{-1} \mathrm{de} \mathrm{NaCl}\right)$} \\
\cline { 2 - 5 } & 0 & 2 & 4 & 6 & 8 \\
\hline Comprimento (mm) & $8,4 \pm 0,3 \mathrm{~b}$ & $10,6 \pm 0,1 \mathrm{a}$ & $9,0 \pm 0,9 \mathrm{ab}$ & $8,2 \pm 1,0 \mathrm{~b}$ & $7,9 \pm 0,9 \mathrm{~b}$ \\
Massa (mg) & $3,7 \pm 0,4 \mathrm{~b}$ & $10,6 \pm 1,3 \mathrm{a}$ & $6,2 \pm 3,1 \mathrm{ab}$ & $3,7 \pm 1,6 \mathrm{~b}$ & $3,9 \pm 2,7 \mathrm{~b}$ \\
Sobrevivência (\%) & $91,3 \pm 6,0 \mathrm{a}$ & $99,0 \pm 1,4 \mathrm{a} 71,3 \pm 2,1 \mathrm{~b}$ & $70,7 \pm 6,6 \mathrm{~b} 38,3 \pm 8,8 \mathrm{c}$ \\
\hline & \\
(1)Médias seguidas de letras iguais, nas linhas, não diferem pelo teste de \\
Tukey, a 5\% de probabilidade.
\end{tabular}

salinidade é uma resposta que varia conforme a espécie, as diferenças genéticas e o estágio do desenvolvimento dos peixes (Boeuf \& Payan, 2001; Fashina-Bombata \& Busari, 2003).

Nas salinidades mais altas, houve mortalidade total das larvas de tambaqui em 16, 20 e 25 horas de exposição a 14,12 e $10 \mathrm{~g} \mathrm{~L}^{-1}$, respectivamente, e após 10,18 e 29 horas, respectivamente, para larvas de apaiari, às mesmas concentrações salinas. Jomori et al. (2012) verificaram tempo de tolerância menor para larvas de pacu, com mortalidade total em 2 horas, após a exposição às salinidades de $12 \mathrm{e} 14 \mathrm{~g} \mathrm{~L}^{-1} \mathrm{e}$, em 8 horas, a $10 \mathrm{~g} \mathrm{~L}^{-1}$. Concentrações salinas não adequadas podem ser prejudiciais tanto para as espécies de peixe de água doce quanto para as marinhas, com efeito direto na alteração do comportamento natatório (Luz et al., 2008; Luz \& Santos, 2008), no comportamento alimentar (ao diminuir o consumo de alimento) e na desidratação muscular (Luz et al., 2008). Entretanto, mesmo que as salinidades altas não sejam ambientes propícios à criação das larvas das espécies estudadas, o conhecimento do tempo de tolerância pode auxiliar em manejos profiláticos e terapêuticos com o uso do sal,

Tabela 2. Valores médios das taxas de sobrevivência (\%) de larvas de matrinxã (Brycon amazonicus), apaiari (Astronotus ocellatus) e piau (Leporinus macrocephalus), ao final do período experimental ${ }^{(1)}$.

\begin{tabular}{|c|c|c|c|}
\hline \multirow[t]{2}{*}{ Tratamento } & \multicolumn{3}{|c|}{ Espécies $^{(2)}$} \\
\hline & Matrinxã & Apaiari & Piau \\
\hline \multicolumn{4}{|l|}{ Salinidade (S) } \\
\hline $\mathrm{S}_{0}$ (água doce) & $57,33 \pm 10,8 b$ & $80,33 \pm 13,4 a$ & $92,00 \pm 8,6 \mathrm{a}$ \\
\hline $\mathrm{S}_{2}(2 \mathrm{~g}$ de $\mathrm{NaCl} \mathrm{L}-1)$ & $79,33 \pm 6,3 \mathrm{a}$ & $88,67 \pm 10,2 \mathrm{a}$ & $92,50 \pm 4,7 \mathrm{a}$ \\
\hline $\mathrm{S}_{4}\left(4 \mathrm{~g}\right.$ de $\left.\mathrm{NaCl} \mathrm{L}^{-1}\right)$ & $53,33 \pm 8,0 \mathrm{~b}$ & $29,17 \pm 23,1 b$ & $91,00 \pm 3,5 \mathrm{a}$ \\
\hline \multicolumn{4}{|c|}{ Quantidade de alimento $(\mathrm{Q})^{(3)}$} \\
\hline $\mathrm{Q}_{1}$ & $62,37 \pm 13,9$ & $72,40 \pm 30,8$ & $90,88 \pm 6,6$ \\
\hline$\underline{\mathrm{Q}_{2}}$ & $64,30 \pm 15,3$ & $59,67 \pm 32,1$ & $92,77 \pm 4,4$ \\
\hline$\overline{\mathrm{S}_{0} \mathrm{Q}_{1}}$ & $56,89 \pm 7,7$ & $87,67 \pm 10,6$ & $90,67 \pm 12,1$ \\
\hline $\mathrm{S}_{0} \mathrm{Q}_{2}$ & $57,78 \pm 15,2$ & $73,00 \pm 14,0$ & $93,33 \pm 5,8$ \\
\hline $\mathrm{S}_{2} \mathrm{Q}_{1}$ & $79,56 \pm 4,1$ & $91,33 \pm 10,9$ & $91,67 \pm 2,9$ \\
\hline $\mathrm{S}_{2} \mathrm{Q}_{2}$ & $79,11 \pm 9,1$ & $86,00 \pm 10,8$ & $93,33 \pm 5,8$ \\
\hline $\mathrm{S}_{4} \mathrm{Q}_{1}$ & $50,67 \pm 2,3$ & $38,33 \pm 30,6$ & $90,33 \pm 4,5$ \\
\hline $\mathrm{S}_{4} \mathrm{Q}_{2}$ & $56,00 \pm 11,6$ & $20,00 \pm 12,1$ & $91,67 \pm 2,9$ \\
\hline
\end{tabular}

${ }^{(1)}$ Médias seguidas de letras iguais, nas colunas, não diferem entre si, pelo teste de Tukey, a 5\% de probabilidade. ${ }^{(2)}$ Período de 5, 10 e 12 dias de alimentação ativa, para larvas de matrinxã, apaiari e piau, respectivamente. ${ }^{(3)}$ Quantidades diárias de náuplios de Artemia: $\mathrm{Q}_{1}=1.250$ e $\mathrm{Q}_{2}=2.500$ náuplios por larva de matrinxã; $\mathrm{Q}_{1}=500, \mathrm{Q}_{2}=1.000$ e $\mathrm{Q}_{1}=2.000$,

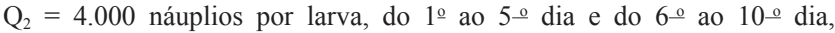
respectivamente, para o apaiari; $\mathrm{Q}_{1}=100$ e $\mathrm{Q}_{2}=200$ náuplios por larva, para os três primeiros dias, seguido de um aumento em dobro a cada três dias, para o piau. 
com banhos de curta duração. Esses tipos de banhos com $\mathrm{NaCl}$, de rápidas imersões, são procedimentos recomendados no combate a várias enfermidades (Pavanelli et al., 1998).

As larvas das espécies estudadas mostraram-se estenoalinas, com limite tolerável de salinidade que, no geral, foi de até $2 \mathrm{~g} \mathrm{~L}^{-1}$ (ou até $4 \mathrm{~g} \mathrm{~L}^{-1}$ para o piau) (Tabela 2). Os teleósteos vivem em ambientes sujeitos a variações de concentrações iônicas, o que favoreceu o desenvolvimento de um mecanismo sofisticado de osmorregulação (Tseng \& Hwang, 2008), e essa evidência é nítida nos peixes eurialinos. Os peixes são capazes de manter a concentração osmótica interna e a homeostase iônica a diferentes gradientes de salinidade, o que permite o funcionamento normal das células e dos sistemas fisiológicos para a sobrevivência das espécies (Hwang \& Lee, 2007). No entanto, há um limite para que a efetividade desse mecanismo de osmorregulação não seja afetado.

$\mathrm{O}$ crescimento dos animais, em comprimento e em massa, também foi influenciado pela salinidade da água. Em geral, o crescimento à salinidade de $2 \mathrm{~g} \mathrm{~L}^{-1}$ foi superior ou semelhante aos obtidos em água doce (Tabelas 1, 3 e 4), exceto em larvas de piau, que apresentaram médias semelhantes também a $4 \mathrm{~g} \mathrm{~L}^{-1}$ (Tabela 5). Em larvas de tambaqui, o crescimento foi maior em água salinizada a $2 \mathrm{~g} \mathrm{~L}^{-1}$, que é 2,8 vezes superior à massa alcançada em água doce, e foi gradativamente menor com o aumento da salinidade de 4 para $8 \mathrm{~g} \mathrm{~L}^{-1}$ (Tabela 1). Essa resposta positiva do crescimento em água levemente salinizada também foi observada em larvas de pacu (Jomori et al., 2012).

Houve efeito associado entre salinidade e quantidade de náuplios de Artemia. As médias de comprimento e a massa foram influenciadas pela interação dos dois

Tabela 3. Desdobramento da interação entre a salinidade e a quantidade de náuplios de Artemia $^{(1)}$, para as médias de comprimento e massa das larvas de matrinxã (Brycon amazonicus), após os primeiros cinco dias de alimentação $\operatorname{ativa}^{(2)}$.

\begin{tabular}{lccccc}
\hline $\begin{array}{l}\text { Salinidade } \\
\left(\mathrm{g} \mathrm{L}^{-1} \mathrm{de} \mathrm{NaCl}\right)\end{array}$ & \multicolumn{2}{c}{ Comprimento total $(\mathrm{mm})$} & & \multicolumn{2}{c}{ Massa $(\mathrm{mg})$} \\
\cline { 2 - 3 } \cline { 5 - 6 } \cline { 5 - 6 }$\left(\mathrm{S}_{0}\right)$ & $\mathrm{Q}_{1}$ & $\mathrm{Q}_{2}$ & & $\mathrm{Q}_{1}$ & $\mathrm{Q}_{2}$ \\
$2\left(\mathrm{~S}_{2}\right)$ & $12,6 \pm 0,2 \mathrm{Aab}$ & $13,4 \pm 0,1 \mathrm{Aa}$ & & $10,8 \pm 0,4 \mathrm{Bab}$ & $13,2 \pm 0,7 \mathrm{Aa}$ \\
$4\left(\mathrm{~S}_{4}\right)$ & $13,4 \pm 0,2 \mathrm{Aa}$ & $13,6 \pm 0,4 \mathrm{Aa}$ & & $12,8 \pm 0,6 \mathrm{Aa}$ & $15,0 \pm 1,1 \mathrm{Aa}$ \\
\hline
\end{tabular}

${ }^{(1)}$ Quantidades diárias de náuplios de Artemia: $\mathrm{Q}_{1}=1.250$ e $\mathrm{Q}_{2}=2.500$ náuplios por larva. ${ }^{(2)}$ Médias seguidas de letras iguais, maiúsculas nas linhas e minúsculas nas colunas, não diferem pelo teste de Tukey, a $5 \%$ de probabilidade. fatores em matrinxã (Tabela 3), apaiari (Tabela 4) e piau (Tabela 5).

Para larvas de matrinxã, a maior quantidade de náuplios $\left(\mathrm{Q}_{2}\right)$ proporcionou um crescimento em massa maior na água doce (Tabela 3). No entanto, em água salinizada, o crescimento foi semelhante, entre os tratamentos, com as duas quantidades de alimento $\left(\mathrm{Q}_{1}\right.$ e $\mathrm{Q}_{2}$ ). Para as larvas desta espécie, o resultado mais positivo foi o aumento da taxa de sobrevivência, que foi de cerca de $79 \%$ a $2 \mathrm{~g} \mathrm{~L}^{-1}$, contra $57 \%$ em água doce (Tabela 2), em razão, provavelmente, da diminuição do canibalismo em consequência da maior oferta de alimento, proporcionada pela disponibilidade contínua dos náuplios de Artemia em ambiente salinizado. Weingartner \& Zaniboni Filho (2004) relataram que, para larvas de Pimelodus maculatus, espécie que também apresenta canibalismo no início da alimentação exógena, a maior sobrevivência observada em água salinizada $\left(2 \mathrm{~g} \mathrm{~L}^{-1}\right)$ pode estar associada à distribuição dos náuplios de Artemia na coluna d'água, pelo aumento do tempo de vida desses organismos.

Tabela 4. Desdobramento da interação entre a salinidade e a quantidade de náuplios de Artemia $^{(1)}$, para as médias de comprimento e massa das larvas de apaiari (Astronotus ocellatus), ao final de 10 dias de criação $^{(2)}$.

\begin{tabular}{lccccc}
\hline Salinidade & \multicolumn{2}{c}{ Comprimento total $(\mathrm{mm})$} & & \multicolumn{2}{c}{ Massa $(\mathrm{mg})$} \\
\cline { 2 - 3 } \cline { 5 - 6 }$\left(\mathrm{g} \mathrm{L}^{-1} \mathrm{NaCl}\right)$ & $\mathrm{Q}_{1}$ & $\mathrm{Q}_{2}$ & & $\mathrm{Q}_{1}$ & $\mathrm{Q}_{2}$ \\
\hline $0\left(\mathrm{~S}_{0}\right)$ & $14,52 \pm 0,7 \mathrm{Aa}$ & $15,47 \pm 0,6 \mathrm{Ab}$ & & $45,26 \pm 8,6 \mathrm{Aa}$ & $53,14 \pm 5,1 \mathrm{Ab}$ \\
$2\left(\mathrm{~S}_{2}\right)$ & $14,32 \pm 0,8 \mathrm{Ba}$ & $18,36 \pm 0,6 \mathrm{Aa}$ & & $43,60 \pm 13,2 \mathrm{Ba}$ & $91,02 \pm 13,5 \mathrm{Aa}$ \\
$4\left(\mathrm{~S}_{4}\right)$ & $10,35 \pm 1,1 \mathrm{Bb}$ & $13,19 \pm 0,8 \mathrm{Ac}$ & & $15,30 \pm 8,3 \mathrm{Ab}$ & $32,90 \pm 8,6 \mathrm{Ab}$ \\
\hline
\end{tabular}

${ }^{(1)}$ Quantidades diárias de náuplios de Artemia: $\mathrm{Q}_{1}=500$ e $\mathrm{Q}_{2}=1.000$ náuplios por larva' durante os primeiros cinco dias; e $\mathrm{Q}_{1}=2.000 \mathrm{e}$ $\mathrm{Q}_{2}=4.000$ náuplios por larva do $6^{\circ}$ ao $10^{\circ}$ dia. ${ }^{(2)}$ Médias seguidas de letras iguais, maiúsculas nas linhas e minúsculas nas colunas, não diferem pelo teste de Tukey, a 5\% de probabilidade.

Tabela 5. Desdobramento da interação entre a salinidade e a quantidade de náuplios de Artemia $^{(1)}$, para as médias de comprimento e massa das larvas de piau (Leporinus macrocephalus), após 12 dias de alimentação ativa ${ }^{(2)}$.

\begin{tabular}{lccccc}
\hline Salinidade & \multicolumn{2}{c}{ Comprimento total $(\mathrm{mm})$} & & \multicolumn{2}{c}{ Massa $(\mathrm{mg})$} \\
\cline { 2 - 3 } \cline { 5 - 6 }$\left(\mathrm{g} \mathrm{L}^{-1} \mathrm{NaCl}\right)$ & $\mathrm{Q}_{1}$ & $\mathrm{Q}_{2}$ & & $\mathrm{Q}_{1}$ & $\mathrm{Q}_{2}$ \\
\hline $0\left(\mathrm{~S}_{0}\right)$ & $11,49 \pm 0,3 \mathrm{Aa}$ & $11,78 \pm 1,2 \mathrm{Ab}$ & & $10,28 \pm 1,2 \mathrm{Aa}$ & $12,12 \pm 3,8 \mathrm{Ab}$ \\
$2\left(\mathrm{~S}_{2}\right)$ & $11,44 \pm 0,5 \mathrm{Ba}$ & $13,02 \pm 0,4 \mathrm{Aab}$ & & $10,03 \pm 0,7 \mathrm{Ba}$ & $19,80 \pm 1,9 \mathrm{Aa}$ \\
$4\left(\mathrm{~S}_{4}\right)$ & $11,45 \pm 0,3 \mathrm{Ba}$ & $13,62 \pm 0,3 \mathrm{Aa}$ & & $10,74 \pm 1,4 \mathrm{Ba}$ & $21,78 \pm 1,5 \mathrm{Aa}$ \\
\hline
\end{tabular}

${ }^{(1)}$ Quantidades diárias de náuplios de Artemia: $\mathrm{Q}_{1}=100$ e $\mathrm{Q}_{2}=200$ náuplios por larva para os três primeiros dias, com aumento em dobro a cada três dias. ${ }^{(2)}$ Médias seguidas de letras iguais, maiúsculas nas linhas e minúsculas nas colunas, não diferem pelo teste de Tukey, a $5 \%$ de probabilidade. 
Em água doce, a menor quantidade de alimento testada $\left(Q_{1}\right)$ foi suficiente para garantir o crescimento das larvas de apaiari e piau, pois as duas quantidades proporcionaram crescimento semelhante mesmo uma tendo o dobro de náuplios da outra (Tabelas 4 e 5). Porém, com a salinização da água a $2 \mathrm{~g} \mathrm{~L}^{-1}$ (para o apaiari e piau) e a $4 \mathrm{~g} \mathrm{~L}^{-1}$ (para o piau), o crescimento foi maior em $\mathrm{Q}_{2}$. A massa média das larvas do tratamento $\mathrm{Q}_{2}$, em água salinizada, foi praticamente o dobro da massa das larvas do $\mathrm{Q}_{1}$, tanto do apaiari como do piau. Possivelmente, os náuplios excedentes de $\mathrm{Q}_{2}$, que não foram consumidos imediatamente, permaneceram vivos na água salinizada e ficaram disponíveis às larvas para serem consumidos posteriormente. Duas observações relevantes foram a presença contínua de náuplios vivos, em suspensão na coluna d'água das unidades experimentais dos ambientes salinizados, e a menor quantidade de detritos (náuplios mortos) acumulados no fundo dos tanques, diferentemente do verificado no tratamento em água doce. Beux \& Zaniboni Filho (2006) observaram diferentes tempos de sobrevida dos náuplios de Artemia, em meios ligeiramente salinizados e de água doce, com o início da mortalidade dos náuplios em água doce após $40 \mathrm{~min}$ de exposição, e em ambiente salinizado a 1,7\%, após duas horas de exposição.

O uso de água ligeiramente salinizada foi benéfico para a larvicultura intensiva do tambaqui, matrinxã, apaiari e piau e pode ser uma técnica vantajosa para a criação de larvas de espécies de água doce que recebem náuplios de Artemia como alimento vivo. Essa vantagem também foi observada em outras espécies, entre as quais o trairão Hoplias lacerdae (Luz \& Portella, 2002), o mandi-amarelo Pimelodus maculatus (Weingartner \& Zaniboni Filho, 2004), o pintado Pseudoplatystoma corruscans (Beux \& Zaniboni Filho, 2007; Santos \& Luz, 2009), o pacamã Lophiosilurus alexandri (Luz \& Santos, 2008; Santos \& Luz, 2009), o cascudo-preto Rhinelepis aspera (Luz \& Santos, 2010) e o curimatã Prochilodus costatus (Santos \& Luz, 2009).

Outros efeitos positivos têm sido associados ao uso da água salinizada quanto ao gasto energético do animal para a regulação iônica e osmótica, que é maior em água doce do que em água salinizada (Riley et al., 2003; Tseng \& Hwang, 2008). Os gastos energéticos despendidos com a flutuabilidade também são reduzidos com a salinidade, em razão do aumento da gravidade específica do meio (Lam \& Sharma, 1985).
Além disso, a salinização do meio contribui para a diminuição das respostas fisiológicas ao estresse, como redução de cortisol circulante nos peixes (Gomes et al., 2003; Luz et al., 2008).

Em suma, a tolerância à salinidade apresentou respostas espécie-específicas, e a água ligeiramente salinizada a $2 \mathrm{~g} \mathrm{~L}^{-1}$ favoreceu positivamente $\mathrm{o}$ crescimentoe a sobrevivência das larvas, provavelmente pela vantagem da disponibilidade contínua dos náuplios vivos nesses ambientes. A salinização da água deve ser cuidadosamente ajustada às espécies e monitorada, para que o crescimento e a sobrevivência dos animais não sejam afetados em razão da faixa de tolerância. A quantidade de náuplios de Artemia foi um aspecto importante, principalmente, como fator de interação com a salinidade, tanto pelos benefícios, ao assegurar um crescimento adequado, como pela possibilidade de diminuir a quantidade de náuplios e otimizar o potencial de crescimento das larvas.

\section{Conclusões}

1. A larvicultura das espécies estenoalinas tambaqui (Colossoma macropomum), matrinxã (Brycon amazonicus), apaiari (Astronotus ocellatus) e piau (Leporinus macrocephalus) pode ser realizada em água levemente salinizada a $2 \mathrm{~g} \mathrm{~L}^{-1}$.

2. O piau suporta salinidade de até $4 \mathrm{~g} \mathrm{~L}^{-1}$, sem prejuízos ao crescimento e sobrevivência.

3. O fator quantidade de alimento interage com o fator concentração salina, e os náuplios de Artemia são mais aproveitados, como alimento vivo, na larvicultura intensiva das espécies tambaqui, matrinxã, apaiari e piau, em água ligeiramente salinizada.

\section{Referências}

ALTINOK, I.; GRIZZLE, J.M. Effects of low salinities on Flavobacterium columnare infection of euryhaline and freshwater stenohaline fish. Journal of Fish Diseases, v.24, p.361-367, 2001. DOI: 10.1046/j.1365-2761.2001.00306.x.

BEUX, L.F.; ZANIBONI FILHO, E. Influência da baixa salinidade na sobrevivência de náuplios de Artemia sp. Boletim do Instituto de Pesca, v.32, p.73-77, 2006.

BEUX, L.F.; ZANIBONI FILHO, F. Survival and the growth of pintado (Pseudoplatystoma corruscans) post-larvae on different salinities. Brazilian Archives of Biology and Technology, v.50, p.821-829, 2007. DOI: 10.1590/S1516-89132007000500010.

BOEUF, G.; PAYAN, P. How should salinity influence fish growth? Comparative Biochemistry and Physiology. Part C: Toxicology 
and Pharmacology, v.130, p.411-423, 2001. DOI: 10.1016/ S1532-0456(01)00268-X.

CONCEIÇÃO, L.E.C.; YÚFERA, M.; MAKRIDIS, P.; MORAIS, S.; DINIS, M.T. Live feeds for early stages of fish rearing. Aquaculture Research, v.41, p.613-640, 2010. DOI: 10.1111/j.1 365-2109.2009.02242.x.

FASHINA-BOMBATA, H.A.; BUSARI, A.N. Influence of salinity on the developmental stages of African catfish Heterobranchus longifilis (Valenciennes, 1840). Aquaculture, v.224, p.213-222, 2003. DOI: 10.1016/S0044-8486(03)00273-4.

FEIDEN, A.; HAYASHI, C.; BOSCOLO, W.R. Desenvolvimento de larvas de surubim-do-iguaçu (Steindachneridion melanodermatum) submetidas a diferentes dietas. Revista Brasileira de Zootecnia, v.35, p.2203-2210, 2006. DOI: 10.1590/ S1516-35982006000800002.

GARCIA, L. de O.; BECKER, A.G.; COPATTI, C.E.; BALDISSEROTTO, B. Salt in the food and water as a supportive therapy for Ichthyophthirius multifilii infestation on silver catfish, Rhamdia quelen, fingerlings. Journal of the World Aquaculture Society, v.38, p.1-11, 2007. DOI: 10.1111/j.1749-7345.2006.000 68.x.

GOMES, L. de C.; ARAUJO-LIMA, C.A.R.M.; ROUBACH, R.; URBINATI, E.C. Avaliação dos efeitos da adição de sal e da densidade no transporte de tambaqui. Pesquisa Agropecuária Brasileira, v.38, p.283-290, 2003. DOI: 10.1590/ S0100-204X2003000200016.

HWANG, P.-P.; LEE, T.-H. New insights into fish ion regulation and mitochondrion-rich cells. Comparative Biochemistry and Physiology. Part A: Molecular and Integrative Physiology, v.148, p.479-497, 2007. DOI: 10.1016/j.cbpa.2007.06.416.

JOMORI, R.K.; CARNEIRO, D.J.; MALHEIROS, E.B.; PORTELLA, M.C. Growth and survival of pacu Piaractus mesopotamicus (Holmberg,1887) juveniles reared in ponds or at different initial larviculture periods indoors. Aquaculture, v.221, p.277-287, 2003. DOI: 10.1016/S0044-8486(03)00069-3.

JOMORI, R.K.; CARNEIRO, D.J.; MARTINS, M.I.E.G.; PORTELLA, M.C. Economic evaluation of Piaractus mesopotamicus juvenile production in different rearing systems. Aquaculture, v.243, p.175-183, 2005. DOI: 10.1016/j. aquaculture.2004.09.034.

JOMORI, R.K.; LUZ, R.K.; PORTELLA, M.C. Effect of salinity on larval rearing of pacu, Piaractus mesopotamicus, a freshwater species. Journal of the World Aquaculture Society, v.43, p.423-432, 2012. DOI: 10.1111/j.1749-7345.2012.00570.x.

KAISER, H.; ENDEMANN, F.; PAULET, T.G. A comparison of artificial and natural foods and their combinations in the rearing of goldfish, Carassius auratus (L.). Aquaculture Research, v.34, p.943-950, 2003. DOI: 10.1046/j.1365-2109.2003.00948.x.

LAM, T.J.; SHARMA, R. Effects of salinity and thyroxine on larval survival, growth and development in the carp, Cyprinus carpio. Aquaculture, v.44, p.201-212, 1985. DOI: 10.1016/0044-8486(85)90244-3.
LUZ, R.K.; MARTÍNEZ-ÁLVAREZ, R.M.; DE PEDRO, N.; DELGADO, M.J. Growth, food intake regulation and metabolic adaptations in goldfish (Carassius auratus) exposed to different salinities. Aquaculture, v.276, p.171-178, 2008. DOI: 10.1016/j. aquaculture.2008.01.042.

LUZ, R.K.; PORTELLA, M.C. Larvicultura de trairão (Hoplias lacerdae) em água doce e água salinizada. Revista Brasileira de Zootecnia, v.31, p.829-834, 2002. DOI: 10.1590/ S1516-35982002000400004.

LUZ, R.K.; SANTOS, J.C.E. dos. Avaliação da tolerância de larvas do pacamã Lophiosilurus alexandri Steindachner, 1877 (Pisces: Siluriformes) a diferentes salinidades. Acta Scientiarum. Biological Science, v.30, p.345-350, 2008.

LUZ, R.K.; SANTOS, J.C.E. dos. Effect of salt addition and feeding frequency on cascudo preto Rhinelepis aspera (Pisces: Loricariidae) larviculture. Journal of Applied Ichthyology, v.26, p.453-455, 2010. DOI: 10.1111/j.1439-0426.2009.01371.x.

PAVANELLI, G.C.; EIRAS, J. da C.; TAKEMOTO, R.M. Doenças de peixes: profilaxia, tratamento e diagnóstico. Maringá: EDUEM, 1998. 264p.

PORTELLA, M.C.; DABROWSKI, K. Diets, physiology, biochemistry and digestive tract development of freshwater fish larvae. In: CYRINO, J.E.P.; BUREAU, D.; KAPOOR, B.G. (Ed.). Feeding and digestive functions of fishes. Enfield: Science Publishers, 2008. p.227-279.

RABE, J.; BROWN, J.A. A pulse feeding strategy for rearing larval fish: an experiment with yellowtail flounder. Aquaculture, v.191, p.289-302, 2000. DOI: 10.1016/S0044-8486(00)00434-8.

RILEY, L.G.; HIRANO, T.; GRAU, E.G. Effects of transfer from seawater to fresh water on the growth hormone/insulin-like growth factor-I axis and prolactin in the tilapia, Oreochromis mossambicus. Comparative Biochemistry and Physiology Part B, v.136, p.647-655, 2003. DOI: 10.1016/S1096-4959(03)00246-X.

SANTOS, J.C.E. dos; LUZ, R.K. Effect of salinity and prey concentrations on Pseudoplatystoma corruscans, Prochilodus costatus and Lophiosilurus alexandri larviculture. Aquaculture, v.287, p.324-328, 2009. DOI: 10.1016/j.aquaculture.2008.10.014.

SOUZA-BASTOS, L.R.; FREIRE, C.A. The handling of salt by the Neotropical cultured freshwater catfish Rhamdia quelen. Aquaculture, v.289, p.167-174, 2009. DOI: 10.1016/j. aquaculture.2009.01.007.

TSENG, Y.-C.; HWANG, P.-P. Some insights into energy metabolism for osmoregulation in fish. Comparative Biochemistry and Physiology, part C, v.148, p.419-429, 2008.

WEINGARTNER, M.; ZANIBONI FILHO, E. Efeito de fatores abióticos na larvicultura de pintado amarelo Pimelodus maculatus (Lacépède, 1803): salinidade e cor de tanque. Acta Scientiarum. Animal Sciences, v.26, p.151-157, 2004. DOI: 10.4025/ actascianimsci.v26i2.1859.

WURTS, W.A. Using salt to reduce handling stress in channel catfish. World Aquaculture, v.26, p.80-81, 1995.

Recebido em 31 de julho de 2011 e aprovado em 2 de julho de 2013

Pesq. agropec. bras., Brasília, v.48, n.8, p.809-815, ago. 2013 DOI: $10.1590 / \mathrm{S} 0100-204 X 2013000800001$ 